\title{
Standortverhältnisse und Morphometrie von Geranium sanguineum L. auf der Combe Martigny \\ im Walliser Rhônetal, Schweiz
}

\author{
J. EiJsink $†$ und H. van GiLs
}

Geobotanisches Institut der Universität Nijmegen, Niederlande

\section{Site Conditions and Morphometry of Geranium sanguineum L. on the Combe Martigny in the Valaisian Part of the Rhône Vally, Switzerland}

\section{Summary}

A significant larger stem height, internode number per stem, ramification number per stem, leaf-tip length, stem number $/ \mathrm{m}^{2}$, leaf area indes $/ \mathrm{m}^{2}$ and stem number per individual plant (polycormon) of Geranium sanguineum have been observed in forbland of the Geranio-Trifolietum alpestris as compared with forest, woodland and grassland. We summarize this in the statement that Geranium sanguineum attains its maximum vitality in forb communities. The highest presence of $G$. sanguineum falls within the same formation according to the referred literature.

The time consuming analysis of air and soil temperature, wind velocity and plant nutrient content within the soil did not bring in the expected decisive site factor(s) in the vegetation catena forest-forbland-grassland. According to earlier "holistic" field observations the lower vitality and lower presence of $G$. sanguineum in forest as compared with forbland may be caused by the light factor. The absence of G. sanguineum in the majority of the dry grasslands in the research area may be due to excessive drainage of the site or/and present or former mowing or grazing.

The higher vitality of $G$. sanguineum within the forb communities has been observed in the forest bordering type. The decisive site factor is here, following Dierschke, the intermittent sunlight along the forest edge.

Guidelines for the classification of the Geranio-Trifolietum alpestris (in Quercetea pubescentipetraeae, Trifolio-Geranietea or Festuco-Brometea) cannot be derived from the sampled microclimatic and edaphic parameters. The statements of both $\mathbf{J}_{\text {AKUCS (1972) and Dierschke (1974) }}$ about this question turned out to be valid locally only.

\section{Einführung}

In zahlreichen Geländebeobachtungen, im Wallis (vaN GILS und KEYsERs 1978) wie anderswo (Literatur zitiert in van GILs und KozŁowsKa 1977), haben wir Geranium sanguineum als außerordentlich polymorphe Sippe kennengelernt. Dadurch hat sich die Frage nach dem Zusammenhang bestimmter morphologischer Merkmale verschiedener $G$. sanguineum-Populationen und ihrer Standortsfaktoren aufgedrängt.

Weitere Zielsetzung des Untersuchungsprogrammes war die mikroklimatologische und edaphische Analyse waldbegrenzender Staudensäume des Brachypodio-Geranion in Relation zu den benachbarten Wäldern und (Halb-) Trockenrasen. Zweimal wurde 
diese modische Frage schon aufgegriffen, jedoch (scheinbar?) widersprechend beantwortet. JAKUCS (1972) Ergebnisse führen zur Schlußfolgerung, daß die Waldsäume mikroklimatisch schon dem Wald, aber edaphisch noch den Rasen näherstehen. Das kann als Bestätigung seiner Klassifikation der xerothermen Säume in der entsprechenden Waldklasse (in diesem Fall: Quercetea pubescenti-petraeae) aufgefaßt werden. Laut Dierschk Geranietea'-Säume des Leine-Werra-Berglandes aber mehr dem des Freilandes als dem des Waldes". Weiterhin betont dieser Autor die ökologischen (und floristischen) Eigentümlichkeiten der Saumgesellschaften, welche eine syntaxonomische Fassung dieser Phytozönosen auf Klassenniveau (,Trifolio-Geranietea") rechtfertigen sollten.

Auf Grund floristischer Kriterien und ihrer Kenntnisse der Standorte haben van Gils und Koztowska (1977) die Staudensäume unter dem Namen BrachypodioGeranion bei den mitteleuropäischen (Halb-) Trockenrasen (Festuco-Brometea, Brachypodietalia) eingestuft. Hieraus läßt sich die These ableiten, daß auch die Brachypodio-Geranion-Staudensäume auf der Combe Martigny mikroklimatisch und edaphisch mehr den Halbtrockenrasen als den Wäldern ähneln.

\section{Einiges über die Geographie des Untersuchungsgebietee}

\subsection{Topographie}

Die Untersuchungslokalitäten liegen (vgl. Abb. 1) alle am Südosthang der Combe Martigny (Wallis, Schweiz) im (sub-)montanen Bereich $(800-1200 \mathrm{~m}$ ), also gerade oberhalb der Rebkulturen, welche dem Hang den Namen "Sommet des Vignes" verliehen haben. Die Combe Martigny ist der Berg, welcher die Rhône zwingt, ihre westliche Strömungsrichtung plötzlich in eine nordwestliche umzuändern. Der Südosthang ist meist steil und besteht in den Untersuchungslokalitäten aus metamorphem Gestein, besonders aus Gneisen. Der Steilheit wegen sind an manchen Stellen Terrassen angelegt worden. Bis etwa $800 \mathrm{~m}$ werden auf diesen Terrassen Weinreben kultiviert. Weiter oben hat man Mähwiesen angelegt, die heute als Sozialbrache z. T. verstaudet und verbuscht sind.

\subsection{Klima}

Innerwallis gehört bekanntlich zu den inneralpinen Trockentälern (BRAUNBlanquet 1961). Die ozeanischen Luftmassen haben, bevor sie das Mittelwallis erreichen, schon den größten Teil ihrer Regenschauer an den Nord-, West- und Südhängen des Alpenmassives verloren. Im Unterwallis macht sich der atlantische Einflu $B$ schon recht bemerkbar, und zwar sowohl in milden Wintertemperaturen als auch in der Humidität. Stromaufwärts des Rhôneknies bei Martigny wird das Klima schlagartig kontinentaler. Die Niederschlagsmenge nimmt ab, und die Differenzen sowohl zwischen Winter- und Sommer-als auch zwischen Tag- und Nachttemperatur sind größer. Hingegen ist die mittlere Jahrestemperatur von Mittelwallis und Unterwallis nahezu gleich. Martigny hat eine Niederschlagsmenge von $771 \mathrm{~mm}$ im Jahr, 


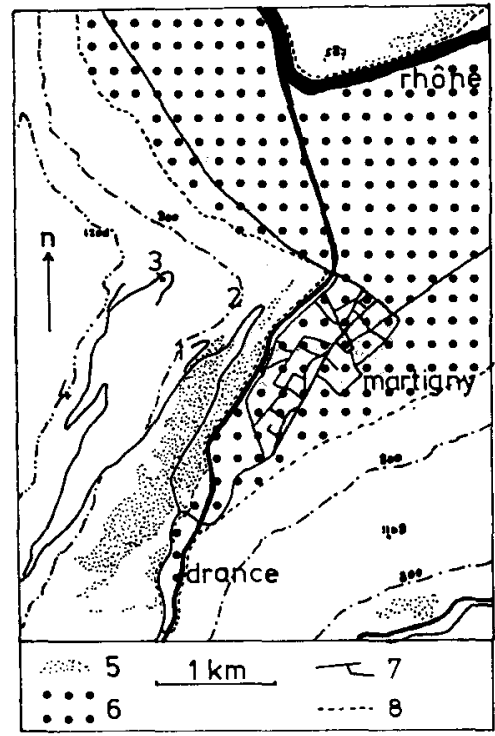

Abb. 1. Das Untersuchungsgebiet. Im Dreieck zwischen 1, 2 und 3 sind die meisten der 54 Kleinquadrate gelegen. 3. Senderturm. 4. Les Rapperins (eine weitere Aufnahmestelle). 5. Weingarten des ,,Sommet des Vignes“. 6. Talboden (liegt bei Martigny auf $\pm 470 \mathrm{~m}$ ). 7. Straßen. 8. Übergang Talboden/Hang.

wogegen Sitten, nur $40 \mathrm{~km}$ weiter stromaufwärts, $500 \mathrm{~mm}$ hat. Das Klimadiagramm der letzteren Ortschaft zeigt schon eine sommerliche Trockenzeit (sensu WaLTER und Lieth). Wie üblich, nimmt die Niederschlagsmenge in höheren Stufen zu, jedoch bleibt sie niedrig, im Vergleich mit Ortschaften gleicher Höhenlage in den Nordalpen. So hat Montana (1453 m), oberhalb von Sitten, nicht mehr als $840 \mathrm{~mm}$ Niederschlag im Jahr. In Zusammenhang mit dieser niedrigen Niederschlagsmenge steht die hohe Zahl der Sonnenscheinstunden und die relativ niedrige Luftfeuchtigkeit. Diese 2 Klimamerkmale gelten wahrscheinlich nicht, oder nur im abgeschwächten Maße, oberhalb $1000 \mathrm{~m}$, wo vor allem in der Nordlage häufig Nebelbänke beobachtet werden.

An Südhängen zwischen $750-1550 \mathrm{~m}$, wo die Geranium sanguineum-Artengruppe optimal vorkommt, liegen die Jahresmittelwerte der Temperatur zwischen $5-8{ }^{\circ} \mathrm{C}$ und die Niederschlagsmengen zwischen $750-1200 \mathrm{~mm}$. Für die Untersuchungslokalitäten darf man die gleichen Werte annehmen.

\subsection{Vegetation}

Wegen der Steilheit der Hänge sind an manchen Stellen Terrassen angelegt. Bis etwa $800 \mathrm{~m}$ werden darauf Weinreben kultiviert. Oberhalb $800 \mathrm{~m}$ sind die Terrassen von Mähwiesen oder deren verbuschten und/oder verstaudeten Brachen eingenommen. An den Steilhängen zwischen den Rebkulturen sind häufig Trockenrasen und kleineres Trockengebüsch anzutreffen. Oberhalb der Weinbaugrenze sind Trocken- 
rasen schon recht selten, die entsprechenden Standorte sind hier von Halbtrockenrasen (Brachypodietalia), z. T. schon mit alpinen Elementen, bewachsen. Außer (Halb-) Trochenrasen besteht die Vegetation des Untersuchungsgebietes aus einem Mosaik von Fettwiesen, Weidebrachen, Ericaceen-Heiden, isolierten Holzgewächsen, Gebüschen und Wäldern (vgl. Abb. 2). Letztere werden abwechselnd von Quercus pubescens, Q. petraea, Picea abies, Larix decidua oder Pinus sylvestris dominiert. Die Eichenwälder sind sicher größtenteils sekundär; als Naturwald kam vom Hangfuß bis zur oberen Grenze des Untersuchungsgebietes, wie man aus Angaben von MExER (1952) ableiten kann, bis zu Mitte des 19. Jahrhunderts ein Lärchenwald mit Buchen, Tannen und eingestreuten Fichten vor. Heute wächst auf gleichem Standort, wenn der anthropogene Standortsfaktor wegfällt, ein primärer Lärchen-Fichtenwald direkt neben einem sekundären Flaumeichenwald. Hieraus und aus dem Vorkommen von G. sanguineum innerhalb Calluna vulgaris-Vaccinium myrtillus-Zwergstrauchheiden läßt sich der Übergangscharakter des Untersuchungsgeländes ableiten.

\section{Methodik}

\subsection{Probeflächen}

Während der ersten Feldarbeitsperiode wurden in etwa $800 \mathrm{~m}$ Seehöhe im Untersuchungsgebiet (Abb. 1) einem Saumweg entlang 54 Kleinquadrate (Datumkode 1 in den Aufnahmen der Bioeinheiten) abgesteckt. Im August wurden dann zur Ergänzung 27 neue Kleinquadrate hinzugefügt (Datumkode 2). Alle Quadrate sind $1 \mathrm{~m}^{2}$ groß. Eine größere Aufnahmefläche würde einen Mangel an Homogenität verursachen. Die Kleinquadrate repräsentieren folgende a priori strukturell verschiedene Vegetationseinheiten einer Xeroserie:

1. Wald (Saponario-Quercetum und Campanulo trachelii-Quercetum BURNAND 1976)
a) geschlossener Wald
b) offener Wald

2. Bandförmige Staudenflur oder Saum (Geranio-Trifolietum alpestris MüLL. 1962)

3. Fleckenförmige Staudenflur (Geranio-Trifolietum alpestris MÜLL. 1962)
a) Staudenflur-Ericaceen-Zwergstrauchheiden (Geranio-Trifolietum genistetosum sagittalis vaN GILs und Keysers 1978)
b) Staudenflur (Geranio-Trifolietum)
c) Staudenflur-Trockenrasen (Geranio-Trifolietum/Bromo-Pulsatilletum BR.-BL. 1961)

4. Trockenrasen (Stipo-Koelerietum valesianae BR.-BL. 1961)
a) Trockenrasen mit Geranium sanguineum
b) Trockenrasen ohne Geranium sanguineum

Die Struktur dieser Bioeinheiten läßt sich aus Abb. 2 und 3, und die floristische Zusammensetzung aus der phytosoziologischen Tabelle ablesen. 


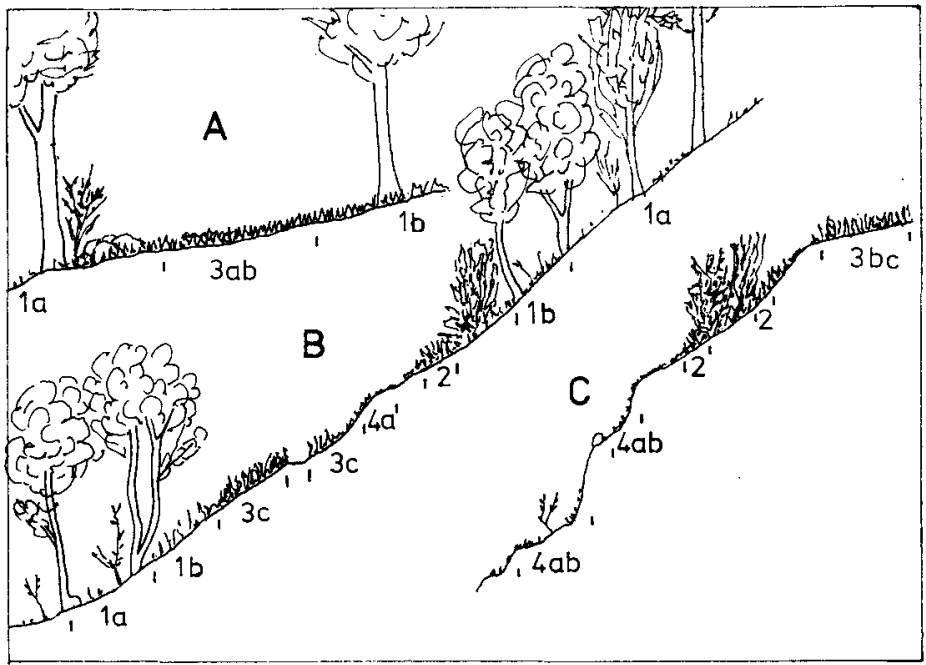

Abb. 2. Verzeichnis im Text. A. Querschnitt des Gebiets zwischen 1000-1200 m im Bereich des Senderturms. B. Querschnitt quer den Saumweg kreuzend auf $\pm 800 \mathrm{~m}$ in der Nähe von Punkt 1 aus der Abb. 1. C. Querschnitt eines Felshanges in der Nähe von Punkt 2 (Abb. 1).

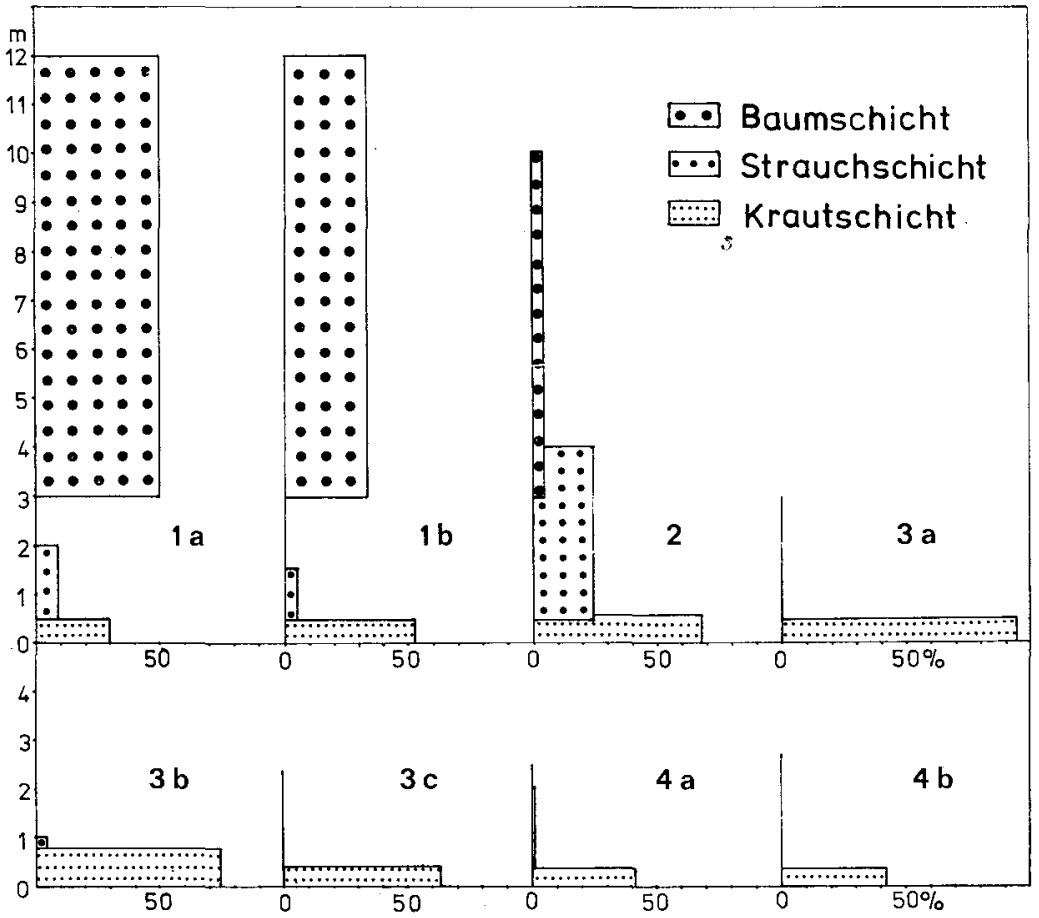

Abb. 3. Schichtung und Deckung in \% der einzelnen Schichten der Bioeinheiten. 
3.2. Biomorphometrie von Geranium sanguineum-Populationen

Neben den Kleinquadraten wurden , at random“ 3-5 Sprosse von $G$. sanguineum gesammelt und getrocknet.

Die Bestimmung der folgenden morphologischen Merkmale sollte uns ermöglichen, ein Bild der morphologischen Variabilität der Geranium sanguineum-Populationen zu entwerfen:

a) Sproßlänge (Abb. 4A)

b) Gliederzahl pro Sproß (Abb. 4B)

c) Zahl der Sproßverzweigungen (Abb. 4C)

d) Quotient, Gliederzahl und Terminalknospenzahl pro Sproß (Abb. 4D)

e) Blattzipfellänge (Abb. 5A); arbiträr wurde für diese Bestimmung der längste Zipfel genommen. Mittelwert dreier Blätter

f) Zipfelzahl pro Blatt (Abb. 5B). Mittelwert dreier Blätter

g) Zahl der Geranium-Sprosse pro Quadratmeter (Abb. 6A)

h) Blattoberfläche in $\mathrm{dm}^{2}$ von $G$. sanguineum pro $\mathrm{m}^{2}$ (Abb. 7)

i) Sproßzahl pro Gruppe (Abb. 6B).

Folgende Annahmen wurden gemacht, um (h) zu berechnen:

- Jedes Sproßglied trägt 2 Blätter.

- Die mittlere Blattgröße ist gleichzusetzen mit der Blattgröße am mittleren Sproßteil.

- Die Blätter haben eine kreisförmige Peripherie.

- Der längste Blattzipfel $\times^{5} / 3$ stellt den Durchschnitt der Blattperipherie dar.

- Die wirkliche Blattoberfläche beträgt $2 / 3$ der Oberfläche innerhalb des Blattumkreises.

\subsection{Makro- und Mikroklima}

\subsubsection{Talboden- und Hangtemperaturen}

Temperaturdifferenzen zwischen dem Talboden und einigen Stellen in einem Hangtranssekt bis auf $1200 \mathrm{~m}$ wurden gelegentlich am Abend mit einem elektrischen Thermometer (Type Wallac) $50 \mathrm{~cm}$ oberhalb der Bodenoberfläche gemessen. Mit einem PKW wurde aus $1200 \mathrm{~m}$ Höhe talwärts gefahren, wobei nach jeweils $100 \mathrm{~m}$ Höhenunterschied angehalten und die Temperatur gemessen wurde.

In der Bioeinheit Wald (800 m) war während der Geländearbeitsperioden ständig ein Thermohygrograph in Betrieb, ein zweiter war in einem Wald am Talboden aufgestellt.

\subsubsection{Temperaturen in den Bioeinheiten}

Zur Bestimmung thermaler Unterschiede zwischen den Bioeinheiten wurden verschiedene komplementäre Verfahren angewandt:

- Eine Temperatursumme wurde nach den Rohrzuckerinversionsmethode von Berthet (1960) bestimmt. In der ersten Geländeperiode wurden dazu 20, in der zweiten 48 Zuckerampullen gleichmäßig über die Bioeinheiten, $5 \mathbf{c m}$ tief im 
Boden, verteilt. Die Meßperiode dauerte 14 Tage. Der $p H$-Wert der Zuckerlösung war mit $\mathrm{HCl}$ auf 2,9 gebracht worden. Die Inversion wurde teilweise direkt an Ort und Stelle gemessen, teilweise wurden die Zuckerlösungen am Ende der Meßperiode mit $\mathrm{KOH}$ neutralisiert und später im Labor gemessen. Mangels eines Temperaturbades im Gelände konnte keine Konstante bestimmt werden, und die angegebenen Inversionswerte bzw. Temperatursummen haben nur einen relativen Wert innerhalb ihrer Meßserie.

- Mit einem elektrischen Thermoanemometer sind kombinierte Boden- und Lufttemperatur-Messungen in den 54 Kleinquadraten durchgeführt worden. Die Lufttemperatur wurde in 5 und $50 \mathrm{~cm}$ Höhe, die Bodentemperatur in $5 \mathrm{~cm}$ Tiefe gemessen, gleichzeitig die Windgeschwindigkeit während \pm 10 Sek. ebenfalls in $50 \mathrm{~cm}$ Höhe und die relative Luftfeuchtigkeit am Boden festgestellt. Eine solche kombinierte Messung dauerte ungefähr 5 Min., was für alle 54 Kleinquadrate \pm 3 Std. bedeutet. In 3 Std. hat sich jedoch die Wetterlage schon so viel geändert, daß die Werte einer Meßserie nicht mehr untereinander verglichen werden können. Deshalb wurden nur einstündige $\left(\mathrm{T}_{\mathrm{x}} \pm \mathbf{3 0}\right.$ Min.) Meßserien zusammengesetzt. Vor dieser Datenverarbeitung hatten wir die Temperaturmessungen arbiträr in 3 Klassen eingeteilt: Klasse $\mathrm{A}: \mathrm{T} \quad 18^{\circ} \mathrm{C}$

$$
\begin{array}{ll}
\mathrm{B}: \mathrm{T} & 18-22{ }^{\circ} \mathrm{C} \\
\mathrm{C}: \mathrm{T} & 22{ }^{\circ} \mathrm{C}
\end{array}
$$

Folgende Datenverarbeitung hat stattgefunden:

$$
\sum_{1-\mathrm{m}}\left\{\frac{\sum_{1-\mathbf{n}} \frac{\mathrm{n}\left(\mathbf{T}_{\mathbf{4 a}}-\mathbf{T}_{\mathrm{x}} \pm 30 \text { Min. }\right)}{\mathrm{m}}}{\mathrm{m}}\right.
$$

$\mathbf{T}=$ Temperatur

$4 \mathrm{a}=$ Jede einzelne Messung der Bioeinheit (4a)

$\mathbf{x}=$ Bioeinheit $(1-4)$

$\mathbf{n}=$ Zahl der Temperaturmessungen innerhalb 30 Min. der $T_{4 a}$

$\mathbf{m}=$ Zahl der mittleren Unterschiede $\mathrm{z}$ wischen den $\mathrm{T}_{4 \mathrm{a}} \cdot$ und $\mathbf{T}_{\mathbf{x}}$-Messungen.

\subsection{Bodenmerkmale}

Im Juni sind neben den 54 Kleinquadraten von zusätzlichen Kleinquadraten der Einheit $(3 \mathrm{~b})$ Bodenproben ausgestochen worden. Von jeder Lokalität wurde dreimal, je auf $5 \mathrm{~cm}$ und $10 \mathrm{~cm}, 70 \mathrm{~cm}^{3}$ Boden entnommen. Die 3 Proben einer Tiefe wurden gemischt. Zwischen Transport und Ionenanalyse wurden die Bodenproben tiefgefroren. Bodenteilchen über $2 \mathrm{~cm}$ wurden von der Ionenanalyse ausgeschlossen. $40 \mathrm{~g}$ der feineren Bodenteilchen wurden abgewogen und mit $\pm 100 \mathrm{~g}$ destilliertem Wasser eine Stunde lang in jodierten Plastikflaschen geschüttelt. Im Filtrat wurden mit einem Autoanalyser $p \mathrm{H}-$ Wert, $\left(\mathrm{NO}_{3}^{-}\right),\left(\mathrm{NH}^{+}\right)$und $\left(\mathrm{PO}_{4}^{---}\right)$gemessen. Der Humusgehalt ist mit dem Gewichtsverlust nach einer 16stündigen Erhitzung bei $375^{\circ} \mathrm{C}$ gleichgesetzt (BALL 1964). 


\subsection{Statistische Auswertung}

Ein einfacher t-Test wurde für den Vergleich der Mittelwerte mit einer Normalverteilung und ähnlichen Standardabweichungen angewandt. In sonstigen Fällen wurde der Mann-Whitney-Rangordnungstest (CAMPBeLL 1967) benutzt (Temperatursumme mittels Rohrzuckerinversion, Geranium sanguineum-Sproßzahl $/ \mathrm{m}^{2}$, Sproßzahl pro Gruppe, $p \mathbf{H}$ der oberen Bodenschicht, Gliederzahl pro Sproß, Blattzipfelzahl or. Blatt). Als signifikant wurden Unterschiede auf der $\mathrm{P}<5 \%$-Stufe betrachtet.

Für die recht komplizierte Verarbeitung der Mikroklimamessungen konnte kein Signifikanztest aufgetrieben werden. Zur Kompensierung sind hier die Anzahl der positiv, negativ und nicht von den $\mathbf{T}_{4 \mathrm{a}}$ abweichenden Messungen wiedergegeben.

Die Bodentaxonomie folgt Kubrëra (1950), die Sippentaxonomie Hess, Landout und Hirzel (1972).

\section{Ergebnis}

\subsection{Biomorphometrie von Geranium sanguineum-Populationen}

Populationen einer Pflanzensippe zeigen oft morphologische Unterschiede, die mit ökologischen Bedingungen zusammenhängen. Dieses Phänomen ist bei Geranium sanguineum besonders ausgeprägt und die Art ist vielleicht deswegen schon früher für ökologisch-morphologische Studien ausgewählt worden (LEWIS 1969; JAKUCS 1972). Das heurige Untersuchungsgebiet eignet sich besonders gut für fortgesetzte Studien in dieser Richtung, da hier eben Geranium sanguineum seine maximale pflanzensoziologische Amplitude aufweist.

Folgende Ergebnisse sind zu diskutieren:

a) Sproßlänge (Abb. 4A)

In Staudenfluren, inklusiv Saum, haben wir jeweils die längeren Sprosse vorgefunden, während sie im Wald und im Trockenrasen kürzer bleiben. Dagegen hat $J_{A K U C S}$ (1972) in der Reihe Wald, Saum, Rasen eine abnehmende Sproßlänge gemessen.

b) Gliederzahl pro Sprosse (Abb. 4B)

Wie zu erwarten war, zeigt die Gliederzahl pro Sprosse eine ähnliche Tendenz wie die Sproßlänge. Die maximale Zahl wird im Saum und sonstigen Staudenfluren erreicht, während die Gliederzahl im Wald und (Halb-) Trockenrasen signifikant niedriger ist.

c) Zahl der Sproßverzweigungen (Abb. 4C)

Die Zahl der Sproßverzweigungen hängt, wie eine vergleichende Betrachtung der Histogramme (Abb. 4 B und 4) zeigt, direkt zusammen mit der Sproßlänge.

d) Längen- versus Breitenwachstum des Sprosses (Abb. 4D)

Als Maß für den Wachstumtypus legen wir den Quotient aus Gliederzahl und Terminalknospenzahl pro Sproß zugrunde. Die Ergebnisse können folgender- 


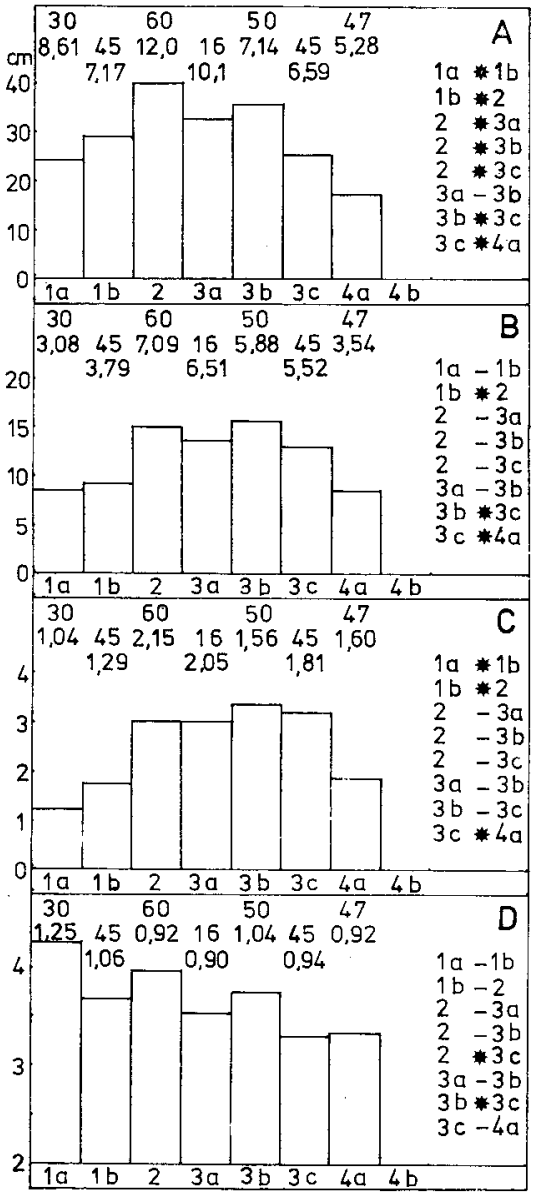

Abb. 4. $\mathbf{A}=$ Mittlere Sproßlänge von Geranium sanguineum. $\mathbf{B}=$ Mittlere Gliederzahl pro Sprosse. $\mathrm{C}=$ Mittlere Zahl der Sproßverzweigungen. D = Mittelwerte der Quotienten der Gliederzahl und Terminalknospenzahl pro Sprosse. Die Zahl der Terminalknospen ist gleich an der Zahl der Verzweigungen +1 .

maßen verbalisiert werden: die Sprosse zeigen im Wald signifikant mehr Längenwachstum als in Staudenfluren, und in letzteren wieder mehr als im Rasen. Im Rasen herrscht bei $G$. sanguineum-Sprossen die Breitenausdehnung schon vor. Das Längen- versus Breitenwachstum hängt wahrscheinlich mit den Lichtverhältnissen zusammen.

e) Blattzipfellänge (Abb. 5 A)

Die größten Blätter kommen in den teilweise beschatteten Saum-Bioeinheiten vor. Noch größere Blätter wären auf Grund der Theorie von LEwIs (1969) in den Wäldern zu erwarten. LewIS Theorie sagt, daß die (genetisch bedingte!) Blattgröße positiv mit der Geschlossenheit des Standorts korreliert. Jedoch in unserem 


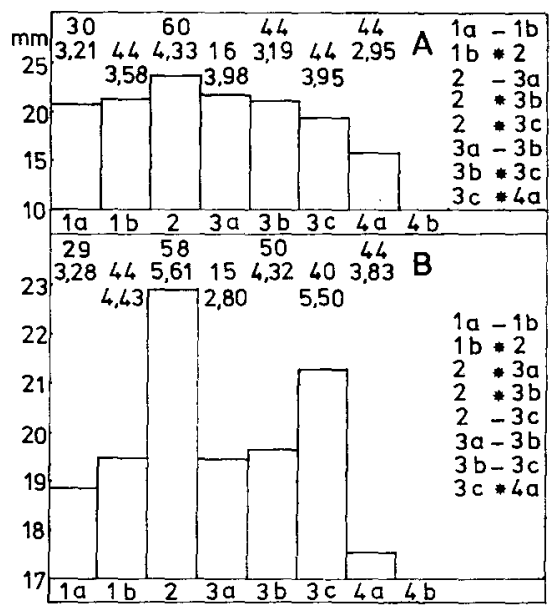

Abb. 5. $A=$ Mittlere Blattzipfellänge. $\mathbf{B}=$ Mittlere Zipfelzahl pro Blatt.
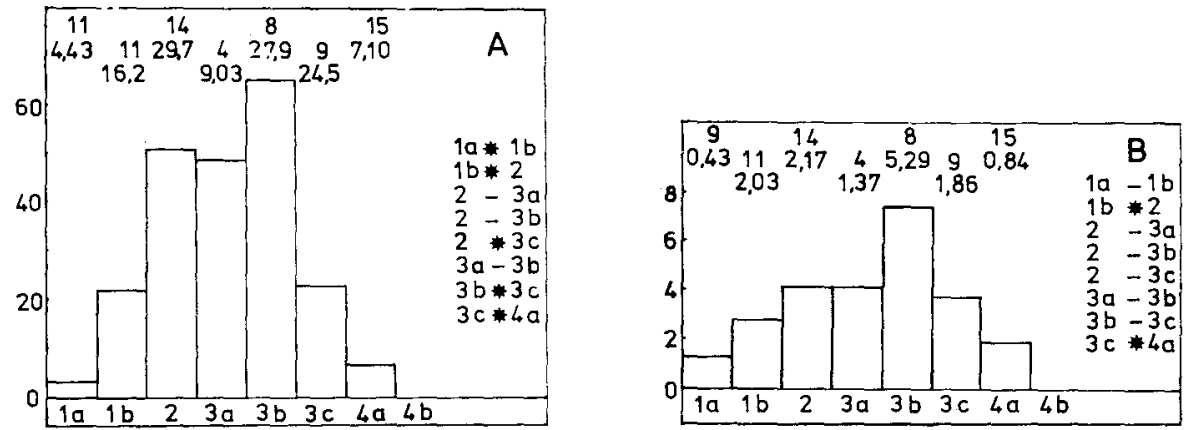

Abb. 6. $A=$ Mittelwert der Zahl der Geranium sanguineum-Sprossen $/ \mathrm{m}^{2}$ in den Bioeinheiten. $\mathbf{B}=$ Mittlere Sprossenzahl pro Gruppe.

Fall sind die Blätter im Wald signifikant kleiner als die des Saumes. Dies kann jedoch nicht als eine Widerlegung LewIs Theorie aufgefaßt werden, da wir nicht kontrolliert haben, ob unsere morphometrischen Unterschiede genetischen Ursprungs oder nur Standortmodifikationen sind.

f) Zipfelzahl pro Blatt (Abb. 5B)

Anstatt des Lewisschen Blattindex haben wir die Zipfelzahl pro Blatt bestimmt. Letztere hängt sowohl mit der Blattgröße wie auch mit der Zipfelbreite zusammen.

g) Zahl der Geranium sanguineum-Sprosse pro Quadratmeter (Abb. 6A)

In Staudenfluren $(2,3 \mathrm{a}, 3 \mathrm{~b})$ ist die Quantität der Geranium-Sprosse signifikant größer als in den sonstigen Bioeinheiten. Dies entspricht den Daten über Sproßlänge und Gliederzahl pro Sproß und bildet somit eine weitere Bestätigung des Staudenfluroptimums von $G$. sanguineum. 


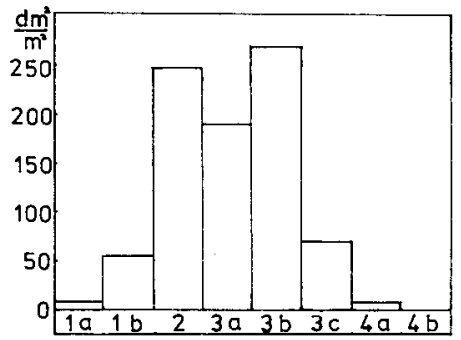

Abb. 7. Gesamtoberfläche der Geranium sanguineum-Blätter $/ \mathrm{m}^{2}$ in jeder Bioeinheit.

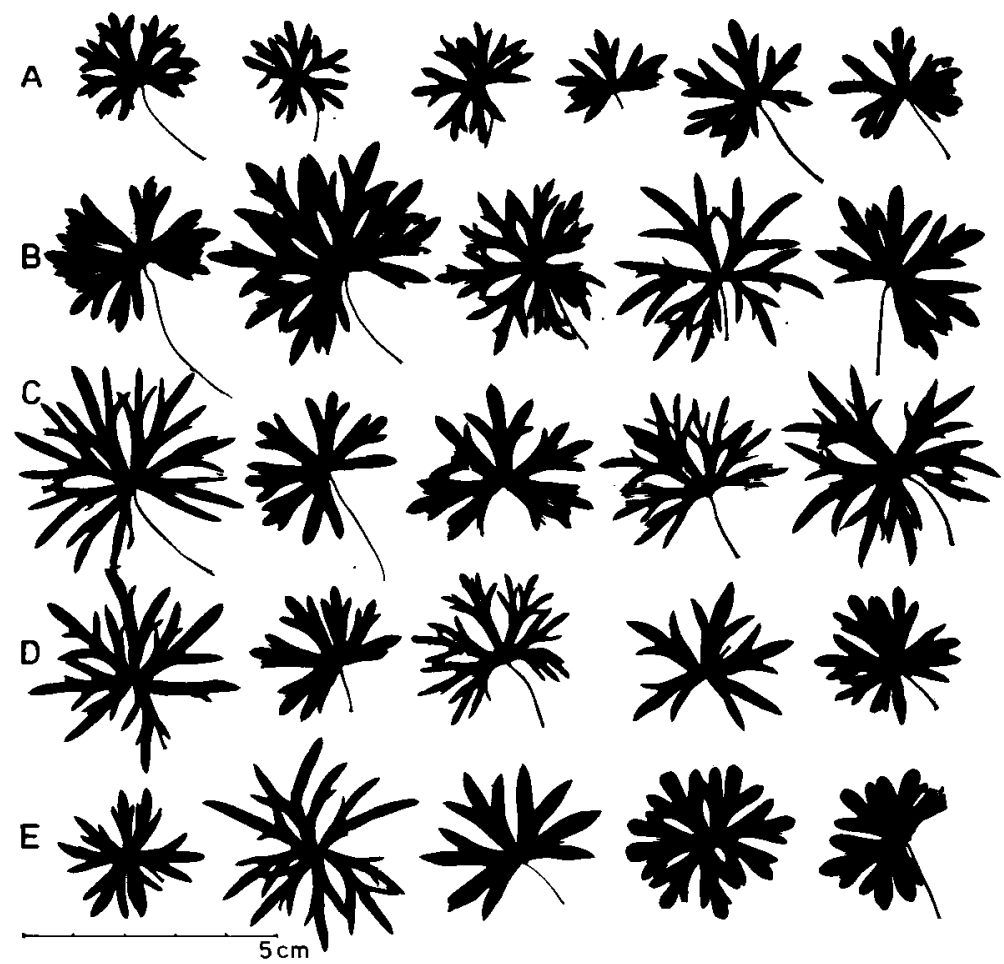

Abb. 8. Einige Blätter von Geranium sanguineum aus dem Untersuchungsgebiet ,,Sommet des Vignes". Es wurde versucht, ein Bild von der Mannigfaltigkeit der vorkommenden Blattformen zu geben. Die Blätter sind nach Größe geordnet.

h) Die Blattoberfläche von G. sanguineum pro Quadratmeter (Abb. 7) gibt am klarsten von allen unserer Parameter eine quantitative Bestätigung des Staudenfluroptimums $(2,3 \mathrm{a}, 3 \mathrm{~b})$ von $G$. sanguineum.

i) Polykormie bei G. sanguineum

Dichtwüchsige, kreisförmige Sproßkolonien sind im Untersuchungsgebiet recht selten. Am häufigsten sind solche noch auf neulich von $G$. sanguineum koloni- 
sierten Straßenrainen, Wiesenbrachen $u$. dgl. beobachtet worden. In allen Fällen handelt es sich um tiefgründige Standorte. Diese Ergebnisse stehen ganz im Gegensatz zu der Aussage von Jakucs (1972), der im Trockenrasen dichte Polykormen nachweist.

Die Sprossenzahl pro Gruppe (Abb. 6B) kann mehr oder weniger gleichgesetzt werden mit der Sproßkoloniedichte sensu JAKUCS (1972). Letzterer verzeichnet eine zunehmende Sproßkoloniedichte von G. sanguineum vom Wald über Saum zum Trockenrasen hin. Unsere Sprossenzahl pro Gruppe hat signifikant die höchsten Werte in Staudensäumen + Staudenfluren (Geranio-Trifolietum alpestris) und nimmt sowohl in Richtung Wald als auch im Trockenrasen ab.

Eine hypothetische Synthese von JaKUOS Beobachtungen über Sproßkolonien und unserer eigenen können uns vielleicht die unterschiedlichen Wetterverhältnisse bieten. Die ungarischen Wetterverhältnisse sind möglicherweise in der vorhergehenden Periode an die Beobachtungsjahre für eine Sproßkoloniebildung von $G$. sanguineum im Trockenrasen günstig gewesen. Im. Walliser Beispiel dagegen ist das Wetter während den letzten Jahren vielleicht ungünstig für eine vegetative Ausbreitung von G. sanguineum in den 'Trockenrasen ausgefallen. Dagegen schaffen hier anthropogene Faktoren, Mähwiesenbrache und Wegebau, wenn auch nur vorïbergehend, Vorbedingungen für eine polykormonale Ausbreitung von $G$. sanguineum.

\subsection{Mesoklima und Mikroklima}

\subsubsection{Mesoklima}

Die Periode August 1975 bis August 1976 war im Wallis, wie mancherorts, sehr trocken, wie die diesbezüglichen monatlichen Niederschlagsmengen für Sitten (vgl. Abb. 9) und Monthey aufweisen. Für die Umgebung Martigny gilt sicher Ähnliches. Möglicherweise haben die Wetterverhältnisse, weil sie die Vegetation beeinflußt haben, einige Tendenzen in Mikroklimaverlauf zwischen den Bioeinheiten abgeändert.

Die Kurven der Thermohygraphen in Bioeinheit (1 a) zeigen, daß die Temperaturen an sonnigen Tagen im Sommer um \pm 13.00 Uhr (MEZ) ein Maximum erreichen

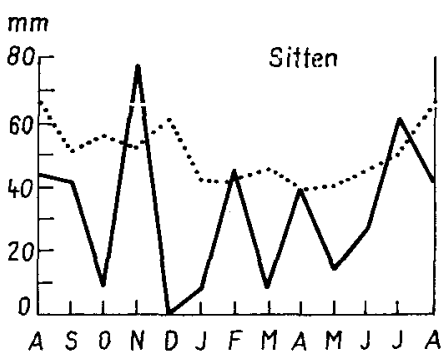

Abb. 9. Monatliche Niederschlagsmengen in Sitten im Zeitraum August 1975 bis August 1976 nach Angaben der Schweizerischen Metereologischen Zentralanstalt, in Vergleich mit den Mittelwerten des Klimadiagramms nach WALTER und JIETH. 
$\left(25-30^{\circ} \mathrm{C}\right)$. Nach diesem Mittagsmaximum kann ein schneller Rückgang bis \pm 17.00 Uhr festgestellt werden, später in eine allmähliche Abnahme bis $\pm 6.00 \mathrm{Uhr}$ übergehend. Die minimale Temperatur betrug $3-10{ }^{\circ} \mathrm{C}$ für alle Bioeinheiten. Ab $6.00 \mathrm{Uhr}$ steigen die Temperaturen schnell an. Die Luftfeuchtigkeit kann an schönen Tagen höchstens bis $35 \%$ abnehmen, nachts liegt sie immer hoch: $80-100 \%$.

Zwischen Talboden $( \pm 500 \mathrm{~m})$ und Montanstufe $( \pm 1200 \mathrm{~m})$ gab es meist keine Temperaturdifferenzen. Wenn es ausnahmsweise Differenzen gab, war der Verlauf unregelmäßig. Erst am späten Abend wurde beim Hinunterfahren eine allmähliche Zunahme der Lufttemperatur um $4-7{ }^{\circ} \mathrm{C}$ festgestellt. Die Minimum- und Maximumwerte am Talboden und am Hang in der Montanstufe sind praktisch gleich, jedoch das Minimum wird am Talboden allmählicher erreicht. Am Abend sind die Temperaturen am Talboden wesentlich höher als am Hang.

\subsubsection{Mikroklima der Bioeinheiten}

Temperatursumme mittels Rohrzuckerinversion

Daß die Temperatursumme im Trockenrasenboden signifikant höher ist als im Wald, darf man ruhig ein triviales Ergebnis nennen (Abb. 10A, B). Die Werte der Säume (2) nehmen genau eine mittlere Stellung ein. Dagegen weichen fleckförmige Staudenfluren und Wälder in ihrer Temperatursumme signifikant ab. Wegen des unterschiedlichen Wetters der beiden Meßperioden können die Messungen nur innerhalb ihrer Periode (A, B) verglichen werden (Abb. 10).

\subsubsection{Temperatur in ${ }^{\circ} \mathrm{C}(\mathrm{Abb} .11)$}

Bei niedriger Lufttemperatur $\left(18^{\circ} \mathrm{C}\right.$ in $50 \mathrm{~m}$ Höhe ü. Boden [H. ü. B.]; Gruppe A) weisen nur die Bodentemperaturen große Unterschiede zwischen den Bioeinheiten auf, während Lufttemperaturen sich nicht deutlich unterscheiden. Die Bodentemperaturen in den Säumen des Geranio-Trifolietum alpestris neigen etwas mehr zum Walde als zum Trockenrasen hin, die der fleckförmigen Staudenfluren der gleichen Assoziation mehr zum Trockenrasen. Auffallend ist, daß in dieser Gruppe A die Windgeschwindigkeit über Trockenrasen $(4 \mathrm{~b})$ relativ hoch und die Luftfeuchtigkeit relativ niedrig ist.

Im mittleren Temperaturbereich $\left(18-22{ }^{\circ} \mathrm{C}\right.$ in $50 \mathrm{~cm}$ [H. ü. B.]; Gruppe B) divergieren die Bodentemperaturen der Bioeinheiten noch stärker, wobei der Waldsaum wie bei der Gruppe A eher waldähnlich ist, die nicht waldbegrenzte Staudenflur aber der Rasentemperatur näherkommt. In 5 und $50 \mathrm{~cm}$ Höhe ergeben sich für alle Bioeinheiten eine gleichwertige Temperatur und relative Luftfeuchtigkeit.

Im Bereich der höheren Temperaturen $\left(22^{\circ} \mathrm{C}\right.$ in $50 \mathrm{~cm}$ [H. ü. B.]; Gruppe C) ist schließlich auch auf 5 und $50 \mathrm{~cm}$ Bestandeshöhe ein Temperaturunterschied zwischen den Bioeinheiten zu erkennen. Der Saum gleicht auf diesen Höhen bei höheren Temperaturen mehr dem Rasen als dem Wald.

Generalisierend können wir sagen, daß die Bodentemperaturen niedriger sind als die Lufttemperaturen, mit Ausnahme der spärlich bewachsenen Kleinquadrate 


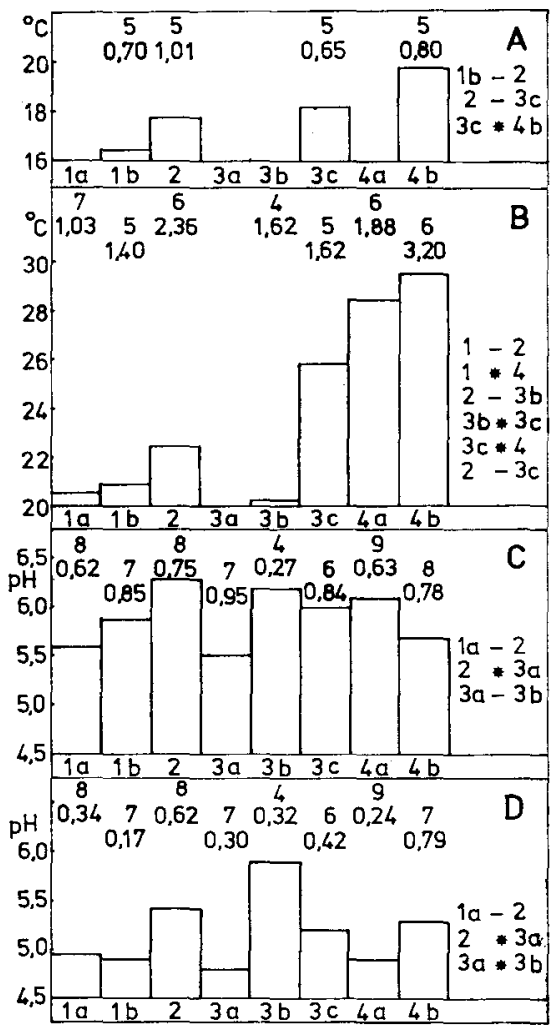

Abb. 10. Temperatursummen mittels der Rohrzuckerinversions-Methode über 14 Tage in Mai und Juni (A) und August (B): $p \mathbf{H}-\mathrm{H}_{2} \mathrm{O}$-Werte in den obersten $5 \mathrm{~cm}$ des Bodens der Kleinquadrate (C) und in $10 \mathrm{~cm}$ Tiefe (D). Oberhalb der Spalten die Zahl der Messungen und die Standarddeviation pro Bioeinheit. Rechts neben der Figur die Ergebnisse der ausgeführten Signifikanzberechnungen: $X=$ signifikante Unterschiede; $-=$ Unterschiede nicht signifikant $(\mathrm{P}<\mathbf{5} \%$-Stufe $)$.

der Trockenrasen (4). In letzteren verursacht die Einstrahlung Bodentemperaturen, welche die der Luft übersteigen.

Die Wälder haben trotz ihres offenen Charakters einen mildernden Einfluß auf die Temperatur (besonders die Bodentemperatur) und die Windgeschwindigkeit.

Die Bioeinheit Saum (2) zeigt in allen 3 Lufttemperaturbereichen (A, B, C) Lufttemperaturen und Bodentemperatursummen, welche mehr mit denjenigen der Wälder übereinstimmen. Die Bioeinheit Staudenflur (3) dagegen ist in dieser Hinsicht immer dem Trockenrasen ähnlicher. In $5 \mathrm{~cm}$ Bestandeshöhe und bei hoher Tagestemperatur ist auch der Saum (2) schon rasenähnlich, was die Temperatursumme angeht. Die Staudenfluren des Geranio-Trifolietum alpestris insgesamt, das heißt die waldbegrenzenden Säume (2) und die waldfernen fleckförmigen Bestände (3) zusammen, stehen zwischen Wald und Trockenrasen, sowohl was die Bodentemperatursumme als auch was die Temperatursumme mittels Rohrzuckerinversion anlangt. Während 


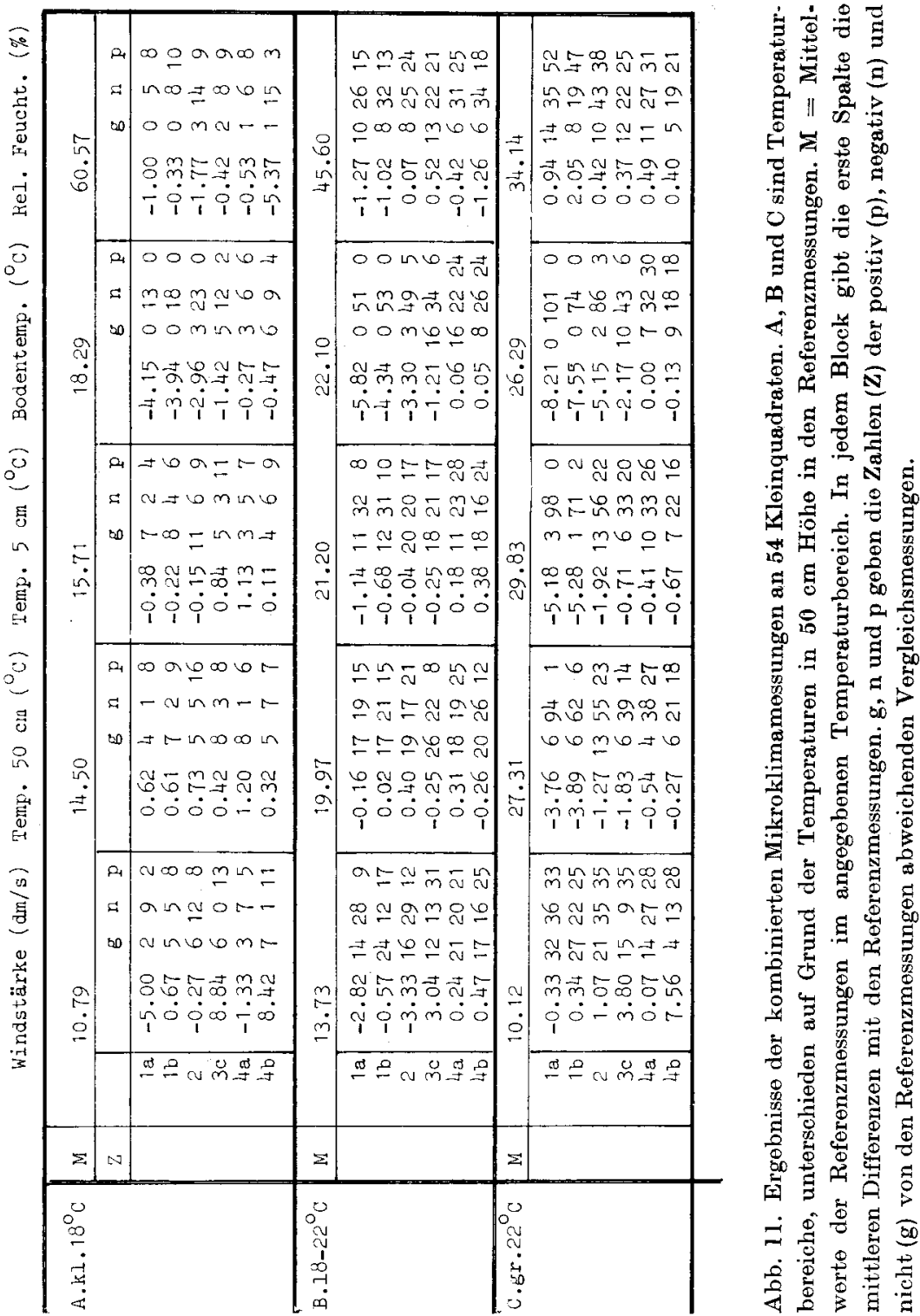




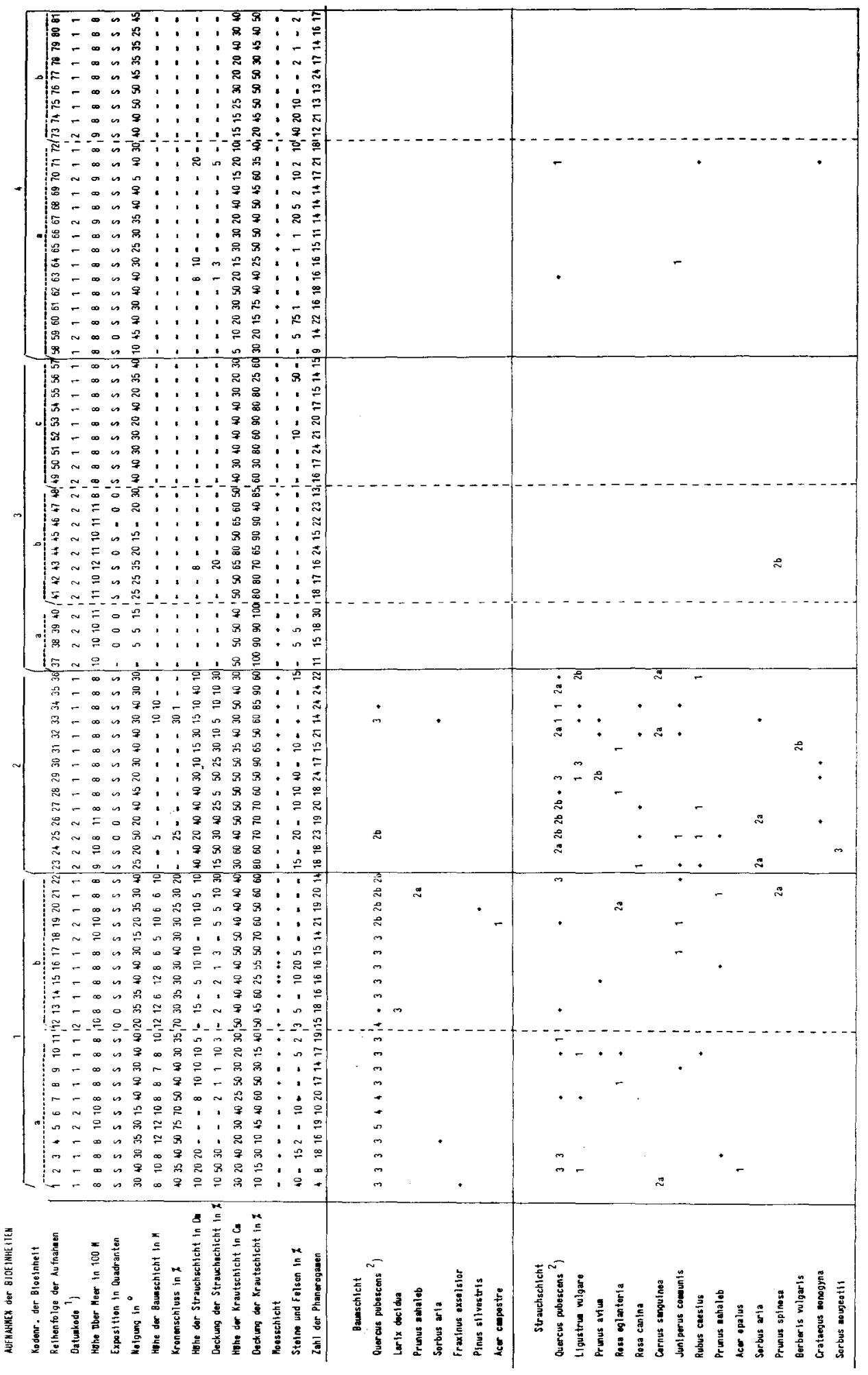




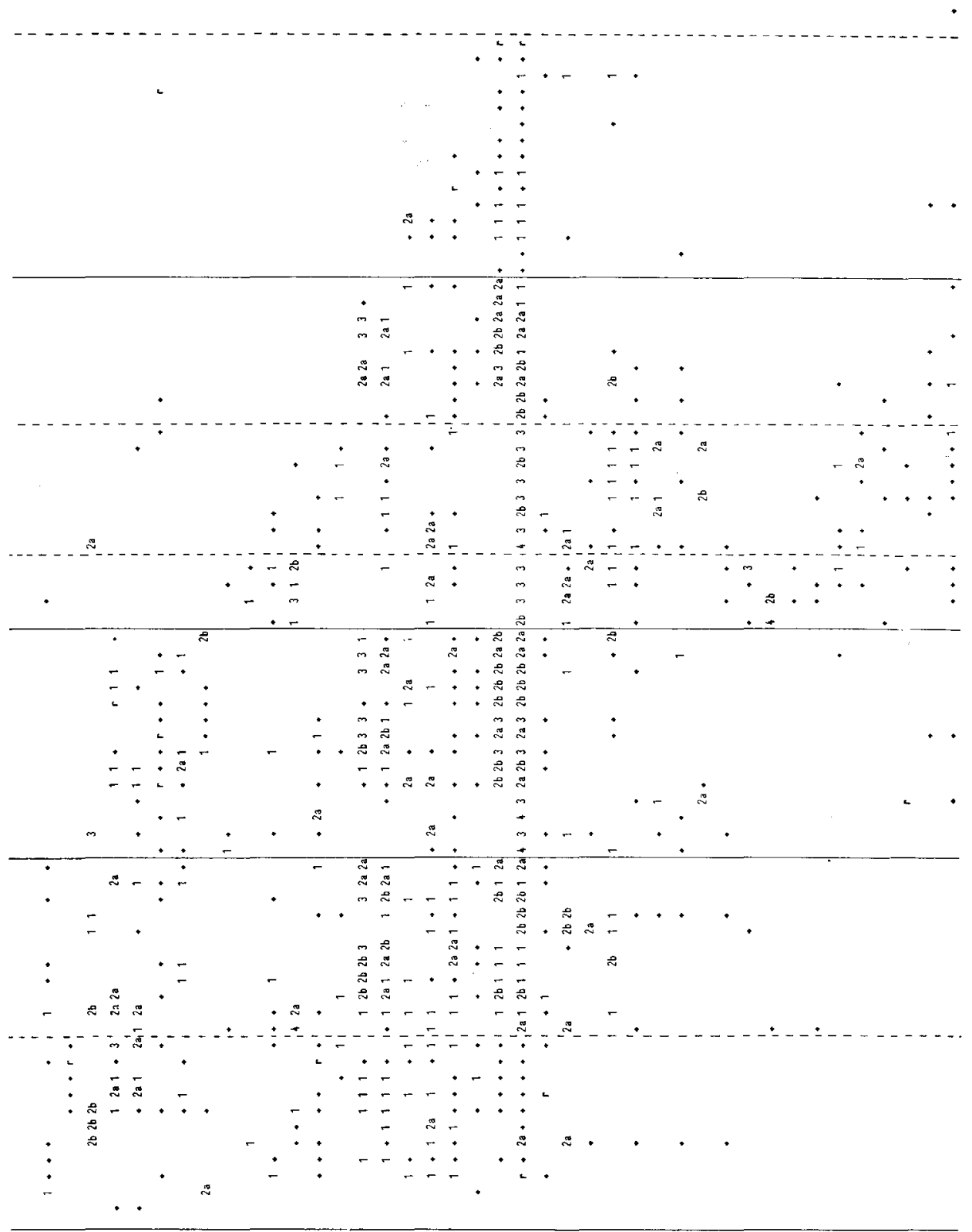

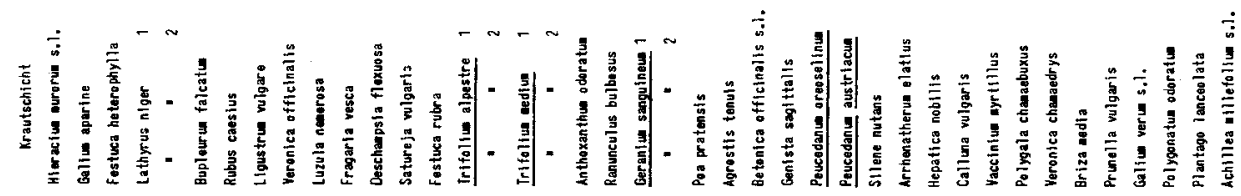
17 Flora, Bd. 168 


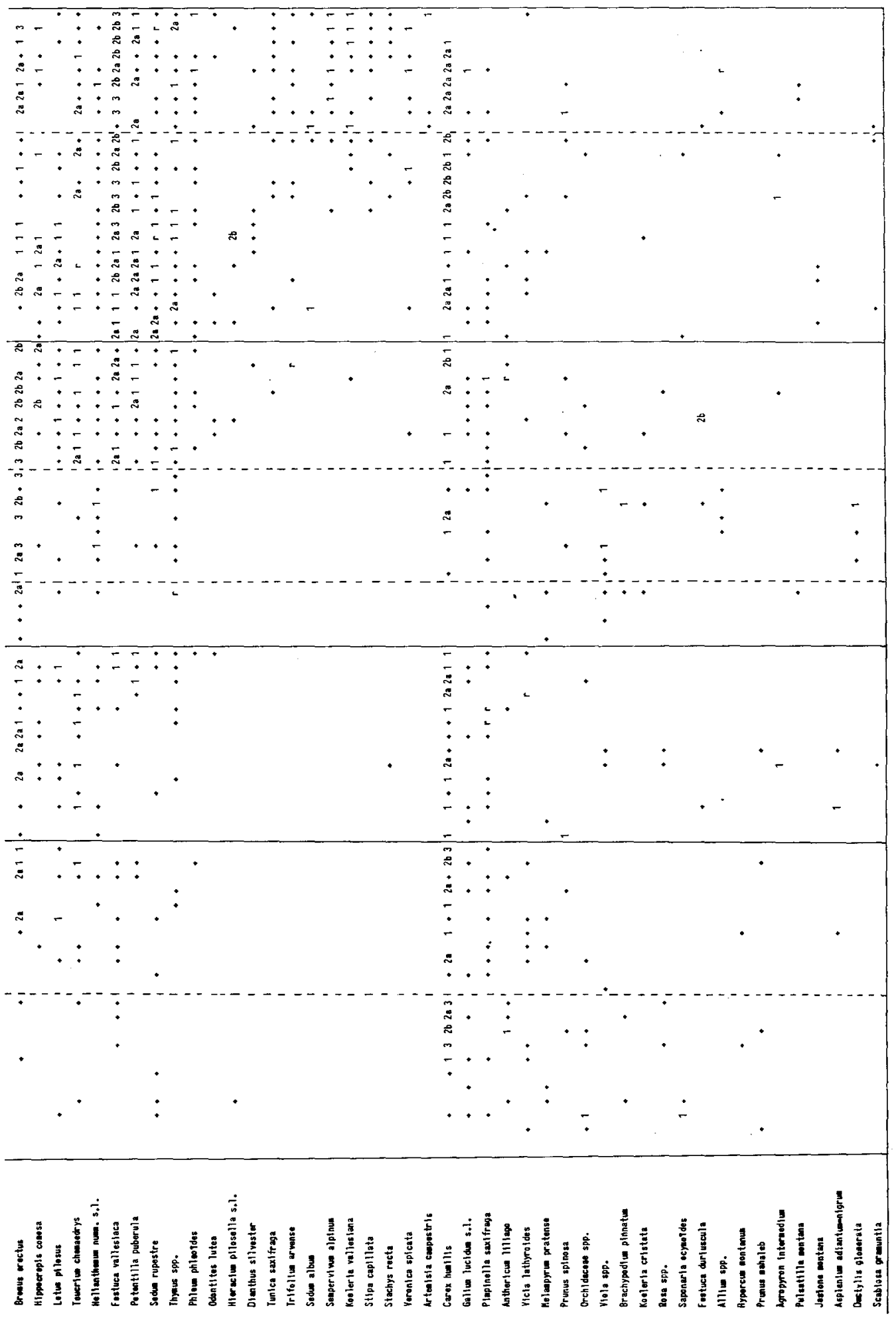


die Temperatursummen des Geranio-Trifolietum alpestris intermediär zwischen Wald und Trockenrasen liegen, umfassen die einzelnen Messungen den ganzen Bereich von Wald- bis zur Rasentemperatur.

In 2 Temperaturbereichen weicht die Windgeschwindigkeit der Bioeinheiten (3c) und $(4 \mathrm{~b})$ stark von den übrigen ab. Im ersteren wächst Geranium sanguineum, so daß im Gebiet auch der Wind kein entscheidender Standortsfaktor für die Präsenz oder Absenz von $G$. sanguineum darstellen kann.

Zwischen 2 Einheiten des Geranio-Trifolietum alpestris (2 und 3c) deuten einige biomorphometrische Parameter, z. B. Blattzipfellänge, Blattoberfläche $/ \mathrm{m}^{2}$, Sproßlänge und Gliederzahl/Terminalknospenzahl, auf eine größere Vitalität von G. sanguineum in waldbegrenzenden Staudenfluren (,Säume“) als in den waldfernen fleckförmigen Staudenfluren. Der entscheidende Standortsfaktor dürfte hier, und wir folgen darin Dienschke (1974), der Halbschatten sein, welcher $u$. a. in den relativ tieferen Bodentemperaturen zum Ausdruck kommt.

\subsubsection{Bodenattribute}

\subsubsection{Bodenmorphologie und Humusgehalt}

Alle Böden unserer Kleinquadrate sind auf Gneisunterlage entstanden. Meistens sind sie flachgründig und der Skelettanteil ist durchwegs sehr hoch.

\section{Tabellenanhang}

Sonstige Arten: Acer campestre (juv.) 11 (r); $16(\mathrm{r}) ;$ Alchemilla vulgaris $40(+) ;$ Anthyllis vulneraria $65(+)$; Asplenium trichomanes $25(+)$; Berberis vulgaris (Juv.) $23(+)$; Carex liparocarpos $67(+)$; Centaurea maculata $73(+)$; Cerastium arvense $13(+) ; 52(+)$; Cotoneaster integerrimus $25(+)$; $26(+) ; 40(+) ;$ Crataegus monogyna $23(+) ; 27(\mathrm{r})$; Cornus sanguinea $1(1) ; 29(+)$; Galium pumi . lum $40(+) ; 41(+) ; 45(+) ; 46(+) ;$ Galium cruciata $44(+)$; Hedera helix $26(+)$; Helictotrichon pratense $46(+)$; Hieracium laevigatum $13(+)$; Hieracium sp. $15(+) ;$ Knautia arvensis $44(+)$; Laserpitium latifolium $26(+) ; 33(\mathbf{r})$; Laserpitium siler $47(+) ;$ Lathyrus sylvestris $25(+)$; Leontodon sp. $40(+)$; Luzula campestris 39(+); 60(+); Melica nutans $25(+) ; 38(+)$; Picea abies (juv.) 5(+); Poa carniolica $73(+)$; Poa bulbosa $62(\mathrm{r})$; Poa annua $7(+)$; Poa nemoralis $4(+)$; Poa concinna $80(+)$; Potentilla erecta $40(+)$; Prunella grandiflora $7(\mathrm{r})$; Prunus avium (juv.) $5(+) ; 29(+) ; 33(+) ; 34(1) ; 53(+) ;$ Prunus mahaleb (juv.) $9(+) ; 21(+) ;$ Primula veris $44(+)$; $46(+)$; Quercus pubescens (juv.) $5(+) ; 22(1) ; 24(+) ; 35(+) ; 56(+) ;$ Rubus idaeus $24(+)$; Rumex acetosa $44(+) ; 47(+)$; Salvia pratensis $53(+)$; Sanguisorba minor $44(+)$; Sedum sexan . gulare $77(+)$; Sedum maximum $25(+) ; 26(+)$; Sempervivum arachnoideum $60(+)$; Senecio doronicum $6(+)$; Solidago virgaurea $50(+)$; Stellaria graminea $50(+)$; Scleranthus annuus $73(+)$; Silene otites $70(+) ; 78(+) ; 79(+)$; Taraxacum sp. $27(\mathbf{r})$; Trisetum flavescens $29(+) ; 46(+)$; $47(1)$; Trifolium repens $52(+)$; Trifolium pratense $35(1)$; Trifolium montanum $48(+)$; Veronica officinalis $23(+) ; 24(+) ; 39(+) ; 50(+) ;$ Vicia onobrychoides $28(2 \mathrm{a}) ; 72(+)$.

Kodierungen in der Tabelle:

1) $1=$ zweimal floristisch inventarisiert, erstmals im Mai-Juni und wiederholt in August; $2=$ nur einmalig (im August) inventarisiert.

2) Wohl nicht nur Quercus pubescens, sondern auch Hybriden dieser Art mit Quercus petraea. 
Im Wald (1) und (Stauden-) Halbtrochenrasen (3c) auf $800 \mathrm{~m}$ herrschen trockene braune Waldböden mit Mullhumus bis auf $10 \mathrm{~cm}$ vor. Beim Trockenrasen (4) kann man meist kaum von Böden sprechen. Das wenige Verwitterungsmaterial ist graugelb und hat nur einen schwach ausgeprägten Mullcharakter. Die Böden der Ericaceenheiden und der Wälder feuchterer Lagen, beide mit $G$. sanguineum, kann man z. T. als Braune Ranker typisieren, teilweise vermitteln sie zwischen diesen und braunen Waldböden. Dort wo der Braune Ranker am klarsten ausgebildet ist (3a und b), ist der Boden bis auf das Muttergestein schwarz. Im Frühling war kaum Rohhumus in den Wäldern vorhanden, und die Bodenaktivität war groß. Beim Fortschreiten des Sommers ließ die Bodenaktivität nach, und es sammelte sich immer mehr Rohhumus an. Angaben über eventuellen. Rohhumus in weniger trockenen Jahren stehen leider nicht zur Verfügung.

Der Humusgehalt der obersten $5 \mathrm{~cm}$ der Böden beträgt in den meisten Bioeinheiten $7,6 \% \pm 1,8$, während sie in der Staudenflur-Ericaceen-Heide $(3 \mathrm{a}, 3 \mathrm{~b}) \mathbf{1 5 , 4} \% \pm$ 7,7 betragen. Die erstgenannten Werte entsprechen der Erwartung für einen braunen Waldboden, sie liegen bedeutend niedriger als der Humusgehalt, den Jakucs in seinen Trockenbuschkomplexen auf Rendzina feststellen konnte.

\subsubsection{Nährstoffgehalt und $p \mathrm{H}$}

Der Nährstoffgehalt der Böden ist niedrig im Vergleich mit den Werten, die JAKUCS (1972) angegeben hat. Die Unterschiede zwischen unseren Bioeinheiten sind unbedeutend. Der Mittelwert für den Gesamtstickstoff $\left(\mathrm{NH}_{4}{ }^{+}+\mathrm{NO}_{2}{ }^{-}+\mathrm{NO}_{3}{ }^{-}\right)$ beträgt $\pm 0,45 \mathrm{mg} \mathrm{N} / 100 \mathrm{~g}$ im Boden der obersten $5 \mathrm{~cm}$ und ist etwas niedriger in $10 \mathrm{~cm}$ Tiefe. Nur in der Staudenflur-Ericaceenheide (3b) sind zweimal erhöhte $\mathrm{N}$. Werte $(0,84$ resp. $0,60 \mathrm{mg} \mathrm{N} / 100 \mathrm{~g}$ Boden) festgestellt worden.

Der Phosphatgehalt der Böden ist ebenfalls niedrig. Im Mittelwert betıägt er 0,10 -0,25 mg P/100 g Boden. Auch in diesem Fall weicht nur die Bioeinheit (3a) mit 0,08 resp. $0,14 \mathrm{mg}$ P/100 g Boden ab. Hier geht es allerdings, im Gegensatz zum N-Gehalt, um einen niedrigeren Wert.

Signifikant abweichende, besonders niedrige $p \mathrm{H}$-Werte (Abb. 4C und D) im Vergleich mit der am meisten verwandten Bioeinheit zeigen nur die Ericaceenheiden mit $G$. sanguineum, wie das schon aus der bloßen Präsenz von Ericaceen abgeleitet werden konnte.

\subsection{Einige phänologische Beobachtungen an Arten der Geranium sanguineum-Artengruppe}

Geranium sanguineum. Ende Mai waren überall im Untersuchungsgebiet die Sprossen fast völlig ausgewachsen, und es setzte die Blütezeit ein, was mit dem Verhalten der Art im Pannonium (JAkucs 1972) übereinstimmt. Im Wald sowie im Trockenrasen blühten nur wenige Exemplare. In den fleckenförmigen Staudenfluren ( $3 \mathrm{c}$ ) waren die Blüten zwar zahlreich, aber sehr klein und lieferten keine Früchte. Das Optimum der Blüte lag in den Säumen. Dort kamen auch gelegentlich Früchte 
vor. Im August wurden keine Blüten mehr angetroffen. Es gab noch keine Anzeichen für eine zweite herbstliche Wuchsperiode, wie JAKucs für G. sanguineum in Trockenrasen gefunden hat.

Sonstige Arten. Laserpitium siler, L. latifolium, Peucedanum austriacum und $P$. oreoselinum wurden nicht blühend angetroffen, was sich wohl mit dem extrem trockenen Sommer und tiefgelegenen Standort exklären läßt. Höher am Hang haben diese Arten 1976 geblüht, und der zweite Autor erinnert sich, 1974 einige blühende $L$. latifolium in etwa $900 \mathrm{~m}$ Höhe bemerkt zu haben. Trifolium alpestre und Trifolium medium verhielten sich wie Geranium sanguineum. Nur wurde die erste der 2 Trifolium-Arten öfter auch im Wald blïhend angetroffen.

\section{Zusammenfassung}

Geranium sanguineum erreicht auf der Combe Martigny in Staudenfluren des Geranio-Trifolietum alpestris eine signifikant größere Sproßlänge, Gliederzahl pro Sproß, Zahl der Sproßverzweigungen, Blattzipfellänge, Sproßzahl $/ \mathrm{m}^{2}$, Blattoberfläche $/ \mathrm{m}^{2}$ und Sproßzahl pro Sproß. kolonie als in Wäldern und Trockenrasen.

Man kann also sagen, daß G. sanguineum in Staudenfluren seine maximale Vitalität erreicht. Aus früheren Arbeiten (1. c.) ist zu entnehmen, daß auch die höchste Präsenz von $G$. sanguineum in die gleiche Formation fällt. Die zeitraubende Analyse der Luft- und Bodentemperatur, Windgesehwindigkeit und Nährstoffionengehalt des Bodens haben uns nicht die erhofften entscheidenden Aufschlüsse über die Standortsfaktoren für die Reihe Wald-Staudenflur-Trockenrasen bringen können. Zurückgreifend auf frühere ,holistische" Geländebeobachtungen möchten wir behaupten, daß für die geringere Vitalität und Präsenz im Wald der Lichtfaktor entscheidend ist, dagegen das Fehlen von $G$. sanguineum in manchen Trockenrasen im Untersuchungsgebiet durch Bodentrockenheit, bedingt durch Exposition, kombiniert mit flachem Profil oder durch gegenwärtige oder ehemalige Mahd und Beweidung bedingt wird. Weiter unten am Hang sowie im sonstigen Mittelwallis kann das Großklima (GILs und KEYSERs 1977) schon als entscheidender Standortsfaktor für die zurücktretende Vitalität und Präsenz von $G$. sanguineum gelten.

Innerhalb der Staudenfluren wird eine größere Vitalität von $G$. sanguineum in den waldbegrenzenden Einheiten festgestellt und nach Dierschke durch den Halbschatten erklärt.

Die mikroklimatologischen und bodenkundlichdn Eregbnisse können keine ökologischen Richtlinien bieten für eine Entscheidung über die Klassifikation des Geranio-Trifolietum alpestris in: Quercetea pubescenti-petraeae, Trifolio-Geranietea oder Festuco-Brometea.

Die Äußerungen von JaKucs (1972) und Dierschke (1974) über letzteres Thema können nach unseren Untersuchungen nur als lokal gültig angesehen werden.

\section{Literatur}

BALL, D. F.: Loss-on-ignition as an estimate of organic matter and organic carbon in non-calcareous soils. J. Soil Science 5, $81-92$ (1964).

Berthet, P.: La mesure écologique de la temperature. Vegetatio 9, $197-207$ (1960).

Braun-Blanquet, J.: Die Inneralpine Trockenvegetation. Geobotanica selecta (ed. R. Tüxen). Fischer, Stuttgart 1961, 273 pp.

Burnand, J.: Quercus pubescens-Wälder und ihre ökologischen Grenzen im Wallis (Zentralalpen). Veröff. Geobot. Inst. E. T. H., Zürich 1976.

Campbeld, R. C.: Statistics for biologists. Cambridge Univ. Press., London 385 (1967). 
DrmRschKe, H.: Saumgesellschaften im Vegetations- und Standortsgefälle an Waldrändern. Scripta Geobot. 6 (1974), $246 \mathrm{pp}$.

GiLs, H. vaN, und Keysers, E.: Saumgesellschaften mit Geranium sanguineum und Trifolium medium in der (Sub)montanen Stufe des Walliser Rhônetals. Folia Geobot. Phytotax. 13 (im Druck).

- and Kozæowska, A. B : Xerothermic forb fringes and forb meadows in the Lublin and Little Poland highlands. Proc. Kon. Ned. Akad, v. Wetenschappen C 80, 281-296 (1978).

Hess, H. E., Landolt, E., und Hirzex, R.: Flora der Schweiz und angrenzender Gebiete. Basel und Stuttgart. 3 Bände (1967, 1970, 1972).

Jakucs, P.: Dynamische Verbindung der Wälder und Rasen. (Quantitative und qualitative Untersuchungen über die synökologischen, phytozönologischen und strukturellen Verhältnisse der Waldsäume.) Budapest 1972.

Kubiëna, W. L.: Bestimmungsbuch und Systematik der Böden Europas. Stattugart-Madrid 1950.

Lewis, M. C.: Genecological differentiation of leaf morphology in Geranium sanguineum L. New Phytol. 68, $481-503$ (1969).

MEYER, K. A.: Frühere Verbreitung der Holzarten und einstige Waldgrenze im Kanton Wallis. III. Im Rhôneknie und Landschaften des rechten Rhôneufers vom Mont Rosel bis Eggerberg. Mitt. Schweiz. Anstalt forstl. Versuchswesen 28, 157-207 (1952).

MüLler, Th.: Die Saumgesellschaften der Klasse Trifolie-Geranietea sanguineï. Mitt. Flor.-soz. Arbeitsgem. N. F. 9, 95- I41 (1962).

Eingegangen 24. November 1977.

Anschriften der Verfasser: Dr. H. vax GILs, T. T. C., 350 Boulevard 1945, Postbus 6, Enschede, Niederlande. 